Service social

\title{
L'informatisation dans les services sociaux ... à un tournant?
}

\section{Martin Poulin}

Volume 36, numéro 1, 1987

L'informatique dans les services sociaux

URI : https://id.erudit.org/iderudit/706337ar

DOI : https://doi.org/10.7202/706337ar

Aller au sommaire du numéro

Éditeur(s)

École de service social de l'Université Laval

ISSN

1708-1734 (numérique)

Découvrir la revue

Citer ce document

Poulin, M. (1987). L'informatisation dans les services sociaux ... à un tournant?

Service social, 36(1), 9-12. https://doi.org/10.7202/706337ar d'utilisation que vous pouvez consulter en ligne.

https://apropos.erudit.org/fr/usagers/politique-dutilisation/ 


\section{AVANT-PROPOS}

\section{L'informatisation dans les services sociaux... à un tournant?}

Le thème de l'informatisation dans les services sociaux peut ne pas paraître prioritaire à certains et pas suffisamment important pour y consacrer un numéro de la revue Service social. Cette première perception peut être reconsidérée si on observe certains facteurs de l'environnement des services sociaux et les milieux de pratique professionnelle, notamment dans plusieurs pays occidentaux.

II faudrait être aveugle pour ne pas voir les changements qui s'opèrent dans l'environnement de la pratique professionnelle ellemême. L'influence des nouvelles technologies et les transformations qu'elles ont suscitées dans de nombreux milieux de travail ne sont pas sans affecter également, à court ou à moyen terme, les milieux de la pratique professionnelle en service social. La technologie micro-informatique est en train de modifier les conditions de travail dans les bureaux. Le milieu des services professionnels y a de plus en plus recours, non plus, comme cela était le cas jusqu'à récemment, pour traiter des masses considérables d'informations, mais pour des opérations spécialisées touchant la nature même du travail professionnel.

Il en est de même dans le milieu du service social. Les applications informatiques qui y avaient cours depuis près de vingt ans étaient principalement axées sur la gestion des services et des ressources (données opérationnelles, finance, paie, ressources matérielles, etc.). Bien que ce type d'applications demeure quantitativement le plus important, d'autres utilisations visent les contenus plus qualitatifs des connaissances et de l'expertise accumulées dans des champs spécifiques d'intervention. Les techniques de l'intelligence artificielle se développent de plus en plus à travers des applications qui touchent l'intervention des professionnels dans ces diverses spécialités. Ces nouveaux instruments sont conçus comme des aides destinées à supporter le professionnel 
dans sa pratique. Cette démarche est récente et constitue un prolongement, dans notre discipline, de développements amorcés dans d'autres champs des sciences humaines, notamment aux États-Unis.

Le mouvement s'est accéléré au cours des dernières années et prend de l'ampleur. Une première conférence internationale (Human System Information Technology Application, "HUSITA-87") regroupant près de deux cent cinquante spécialistes provenant de vingt-deux pays avait lieu en septembre 1987, à Birmingham (Angleterre), sur le thème des "Applications informatiques en service social et dans les professions connexes ». La très grande majorité des participants provenaient de la discipline du service social et ouvraient dans les services ou le milieu universitaire. Cet événement à lui seul témoigne de l'actualité du sujet et constitue une preuve que la recherche dans ce champ d'activités n'en est qu'à ses débuts.

Le milieu francophone est malheureusement quasi absent de cette recherche. Nous avons donc voulu, par ce numéro, attirer l'attention du service social francophone sur ces développements nouveaux et susciter une interrogation au sujet de leur signification pour l'avenir. Dans ce sens, les articles qui suivent jettent un regard sur le passé en essayant d'identifier comment est vu, par les intervenants sociaux, le processus d'informatisation qu'ils ont connu dans leur milieu; en même temps, nous jetons un regard vers le futur en tentant d'explorer certains résultats de recherche sur des projets d'avant-garde.

Dans ce numéro, nous avons voulu présenter une vue d'ensemble de la situation de l'informatisation des services sociaux, principalement par le relevé des résultats de recherches récentes ou en cours dans le domaine, tant au Québec que dans le reste du Canada. Des réflexions plus globales sur la signification et la portée des changements technologiques, pour la société et notre discipline, complètent ce numéro de la revue.

Plus spécifiquement, le premier texte présente une vision des attitudes des intervenants sociaux du Québec au sujet de l'utilisation de l'informatique dans leur milieu de travail. II s'agit des résultats d'une recherche menée en 1986-1987 auprès des praticiens et des cadres ouvrant dans des centres de services sociaux et des centres locaux de services communautaires. Ces résultats donnent un aperçu de leurs attitudes actuelles qui, dans l'ensemble, paraissent favorables bien qu'on distingue des écarts appréciables entre certains groupes d'intervenants selon, entre autres, leur niveau hiérarchique ou leur degré de contact avec l'informatique.

Un deuxième article vient compléter le premier par le fait qu'il rapporte également les résultats d'une recherche effectuée en Ontario en rapport avec le processus d'informatisation en cours dans plusieurs 
agences de service social de la région métropolitaine de Toronto. Cette recherche, tout en étant centrée sur le processus d'implantation, rejoint en partie celle faite au Québec, car elle nous apporte des informations sur les réactions des intervenants et des autres employés qui œuvrent dans ces organismes. L'étude menée par les professeurs Gandy et Djao permet de percevoir le processus dynamique d'informatisation dans certaines agences ontariennes de service social.

Une étude plus restreinte, effectuée auprès des membres de la Corporation des travailleurs sociaux (région administrative de Québec), vient préciser la perception des professionnels du service social au sujet de l'utilisation et de la pertinence de l'informatique dans leur milieu de pratique. Les auteures, Béliveau et Bergeron, tracent un portrait des résultats obtenus lors de cette recherche. Les données de l'enquête laissent croire que les travailleurs sociaux perçoivent assez positivement l'utilisation de l'informatique, et même seraient d'accord à ce qu'on lui fasse une place plus importante, tant dans la gestion que dans la pratique professionnelle.

Deux autres démarches d'informatisation sont réalisées dans deux provinces distinctes mais dans des contextes de pratique semblables. II s'agit de services sociaux en milieu hospitalier pour enfants: I'Hôpital Sainte-Justine, à Montréal, et le Alberta Children's Hospital, à Calgary. Les deux expérimentations en sont cependant à des étapes très différentes et leur nature les distingue nettement.

Au Alberta Children's Hospital, il s'agit d'un projet pilote qui a pour but de développer une application de l'intelligence artificielle à des interventions cliniques effectuées auprès des familles par le Département de service social. En cours de réalisation, ce projet constitue une recherche de pointe en matière de technologie de l'intelligence artificielle appliquée à la pratique professionnelle dans les services sociaux. Gripton, Licker et de Groot font état des méthodes et des principaux concepts utilisés dans cette recherche. Ils ne manquent pas de faire voir l'ampleur de la tâche et les difficultés que pose une telle entreprise.

Du côté québécois, la démarche commencée à l'Hôpital SainteJustine n'est pas non plus sans intérêt. Les praticiennes Lalande-Gendreau et Turgeon-Krawczuk abordent plus précisément le problème de l'élaboration et de la mise en application d'un logiciel informatique destiné à traduire les informations cliniques recueillies par les travailleurs sociaux dans le dossier médical. Cette démarche comporte des exigences de systématisation et de synthèse du processus d'intervention, incluant toutes les étapes, à partir de l'étude de la situation de la famille jusqu'aux recommandations de traitement. Les difficultés rencontrées et les perspectives intéressantes qu'offre le développement d'un tel instrument sont également abordées. 
Les deux derniers articles traitent de thèmes plus larges. Lalande compare l'approche utilisée dans la conception et l'implantation de l'informatisation dans deux établissements du réseau des services sociaux québécois : un centre local de services communautaires et un centre de service sociaux. La comparaison tente de faire ressortir les philosophies qui président à la conception des systèmes informatisés, à la lumière de documents de planification conçus et véhiculés dans ces établissements.

Une dernière présentation discute le bien-fondé et la valeur des changements apportés par la technologie pour la société et l'impact que son usage peut avoir sur la pratique du service social, plus particulièrement sur les couches de population plus défavorisées. Cette réflexion sur les enjeux de l'informatisation et sur sa signification pour les classes sociales défavorisées apporte un éclairage critique et suscite une interrogation importante pour l'avenir de la discipline du service social et de son rapport avec ces clientèles.

Le numéro comporte de plus deux recensions en relation immédiate avec le thème. La première concerne le volume de Schwartz sur l'utilisation de l'ordinateur dans la pratique clinique et ses applications en psychothérapie et en santé mentale. La seconde fait une présentation de la revue Computers in Human Services.

Nous osons espérer que ce numéro sur l'informatisation dans les services sociaux sera de nature à vous intéresser, et surtout à vous interroger sur le tournant que s'apprête à faire le champ du service social en matière d'informatisation, du moins dans les pays occidentaux et anglophones surtout. Quel rôle sommes-nous disposés à jouer dans ce mouvement? passif, comme observateurs étrangers ? ou significatif, en participant de façon active à sa conception de façon à influencer son orientation et à mieux contrôler ses effets sur la pratique de la discipline du service social?

École de service social,

Martin PouliN

Université Laval. 